\title{
Broadband quantum noise reduction in future long baseline gravitational-wave detectors via EPR entanglement
}

\author{
Jacob L. Beckey®, ${ }^{1}$ Yiqiu Ma, ${ }^{2,3}$ Vincent Boyer®, ${ }^{1}$ and Haixing Miao ${ }^{4}$ \\ ${ }^{1}$ School of Physics and Astronomy, University of Birmingham, Birmingham B15 2TT, United Kingdom \\ ${ }^{2}$ Center for Gravitational Experiment, School of Physics, Huazhong University of Science and Technology, \\ Wuhan 430074, China \\ ${ }^{3}$ Theoretical Astrophysics 350-17, Californian Institute of Technology, Pasadena, Californian 91125, USA \\ ${ }^{4}$ School of Physics and Astronomy and Institute of Gravitational Wave Astronomy, \\ University of Birmingham, Birmingham B15 2TT, United Kingdom
}

(Received 20 September 2019; published 14 October 2019)

\begin{abstract}
Broadband quantum noise reduction can be achieved in gravitational-wave detectors by injecting frequency-dependent squeezed light into the dark port of the interferometer. This frequency-dependent squeezing can be generated by combining squeezed light with external filter cavities. However, in future long baseline interferometers (LBIs), the filter cavity required to achieve the broadband squeezing has a low bandwidth — necessitating a very long cavity to mitigate the issue from optical loss. It has been shown recently that by taking advantage of Einstein-Podolsky-Rosen (EPR) entanglement in the squeezed light source, the interferometer can simultaneously act as a detector and a filter cavity. This is an attractive broadband squeezing scheme for LBIs because the length requirement for the filter cavity is naturally satisfied by the length of the interferometer arms. In this paper we present a systematic way of finding the working points for this broadband squeezing scheme in LBIs. We also show that in LBIs, the EPR scheme achieves nearly perfect ellipse rotation as compared to 4-km interferometers which have appreciable error around the intermediate frequency. Finally, we show that an approximation for the optomechanical coupling constant in the 4-km case can break down for longer baselines. These results are applicable to future detectors such as the 10-km Einstein Telescope and the 40-km Cosmic Explorer.
\end{abstract}

DOI: $10.1103 /$ PhysRevD.100.083011

\section{INTRODUCTION}

Gravitational-wave (GW) detectors including LIGO and VIRGO, which recently made breakthrough discoveries, are Michelson-type interferometers with $\mathrm{km}$ size arms [1,2]. They are among the largest and most sensitive experiments humans have ever constructed. To push the limits of scientific discovery even further, larger, more sensitive instruments are already being planned. Two such detectors are the 10-km Einstein Telescope (ET) [3] and the 40-km Cosmic Explorer [4]. They differ from LIGO in many ways, including scale and configuration, but for our purposes, they can be treated in a very similar way mathematically.

All ground-based GW detectors are plagued by various noise sources that result from the fact that they are on Earth (e.g., seismic activity). Once these and all other classical noise sources are suppressed, the sensitivity of GW detectors is ultimately limited by the quantum nature of light. The quantized electromagnetic field is analogous to a quantum harmonic oscillator (position and momentum of a mass are replaced by amplitude and phase quadrature of light). The uncertainty in the amplitude and phase quadratures (quantum fluctuations) limits the sensitivity of interferometric measurements.
The two primary noise sources in GW interferometers are radiation-pressure noise and photon shot noise. The former is due to the quantum fluctuations in the amplitude that cause random fluctuations in the radiation pressure force on the mirrors. The latter is due to the uncertainty in the phase of the light which manifests itself as the statistical arrival time of photons. These noise sources are modeled as entering through the dark port of the interferometer and coupling to the coherent laser light. The detector sensitivity achieved when these uncorrelated (random) fluctuations enter the interferometer is called the standard quantum limit (SQL). It has been shown that this limit could be surpassed if a squeezed vacuum was injected into the interferometer's dark port instead $[5,6]$. Depending on the squeezing angle of the injected squeezed vacuum (see Fig. 1), we can decrease the amplitude or phase fluctuations that limit the detector sensitivity. Amplitude and phase quadratures are conjugate variables (like position and momentum); thus the Heisenberg uncertainty principle states that the product of their uncertainties must be greater than some constant. Thus, by decreasing phase fluctuations, we suffer an increase in amplitude fluctuations. This would not be a problem if at low gravitational-wave frequencies, the mirror 


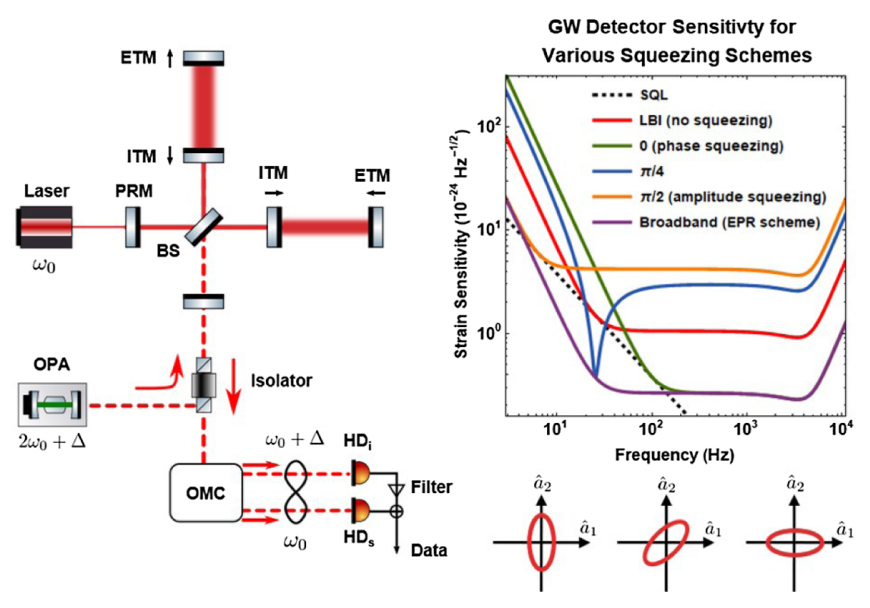

FIG. 1. LBI setup and sensitivity curves for various squeezing schemes. A fixed squeezing angle only surpasses the SQL (black dotted line) over a narrow frequency band. Our broadband squeezing scheme (purple curve) is achieved by rotating the noise ellipse in a frequency-dependent way as shown below the plot. Acronyms used are as follows: end test mirror (ETM), input test mirror (ITM), power recycling mirror (PRM), signal recycling mirror (SRM), and output mode cleaner (OMC).

suspension systems did not have mechanical resonances that amplify these fluctuations and make radiation pressure noise the limiting noise source. Put simply, at low frequencies we need amplitude-squeezed vacuum injection. Once far away from these resonances (at higher frequencies), the detector is then limited by shot noise, and thus a phasesqueezed vacuum is needed. It has been known for some time that frequency-dependent squeezing would allow one to surpass the SQL over all frequencies [7]. These proposals require additional low-loss filter cavities. It was shown recently that an alternative approach to achieving frequency-dependent squeezing without additional cavities is to use EPR-entangled signal and idler beams (different frequency components in a conventional squeezed light source) [8], which has been demonstrated in proof-ofprinciple experiments $[9,10]$. In this paper, we present a systematic way of finding the working points for this broadband squeezing scheme in LBIs. We also show that in LBIs, the EPR scheme achieves nearly perfect ellipse rotation as compared to 4- $\mathrm{km}$ interferometers which have appreciable error. Finally, we show that an approximation for the optomechanical coupling constant in the 4-km case can break down for longer baselines.

\section{THEORY}

\section{A. EPR entanglement in squeezed-light source}

In this section, we illustrate the EPR entanglement generated in the squeezed-light source, which consists of a nondegenerate optical parametric amplifier with a nonlinear electric susceptibility $\left(\chi^{(2)}\right.$ in our case). Such a device takes in two modes and a pump beam (energy source) and produces two amplified modes which we call the signal and idler beams. In frequency space, we can visualize the optical parametric amplifier taking in uncorrelated sidebands and entangling (correlating) them. In fact, any frequency modes $\omega_{1}, \omega_{2}$ within the squeezing bandwidth that suffice $\omega_{p}=\omega_{1}+\omega_{2}$ will be entangled with each other. In our proposed scheme $[8,11]$, we detune the pump field by an amount $\Delta$ (of order $\mathrm{MHz}$ ) such that $\omega_{p}=2 \omega_{0}+\Delta$, where $\omega_{0}$ is the interferometer carrier frequency. This creates correlated sidebands around the frequencies $\omega_{0}$ and $\omega_{0}+\Delta$ (Fig. 2). The corresponding amplitude and phase quadratures defined with respect to $\omega_{0}$ and $\omega_{0}+\Delta$ are therefore entangled $[12,13]$.

In Caves and Schumaker's two-photon formalism [14], the amplitude and phase quadratures are written in terms of sidebands.

$$
\begin{gathered}
\hat{a}_{1}(\Omega)=\frac{\hat{a}\left(\omega_{0}+\Omega\right)+\hat{a}^{\dagger}\left(\omega_{0}-\Omega\right)}{\sqrt{2}}, \\
\hat{a}_{2}(\Omega)=\frac{\hat{a}\left(\omega_{0}+\Omega\right)-\hat{a}^{\dagger}\left(\omega_{0}-\Omega\right)}{i \sqrt{2}}, \\
\hat{b}_{1}(\Omega)=\frac{\hat{b}\left(\omega_{0}+\Delta+\Omega\right)+\hat{b}^{\dagger}\left(\omega_{0}+\Delta-\Omega\right)}{\sqrt{2}}, \\
\hat{b}_{2}(\Omega)=\frac{\hat{b}\left(\omega_{0}+\Delta+\Omega\right)-\hat{b}^{\dagger}\left(\omega_{0}+\Delta-\Omega\right)}{i \sqrt{2}} .
\end{gathered}
$$

The general quadratures for the signal and idler beams can then be written as

$$
\begin{aligned}
& \hat{a}_{\theta}=\hat{a}_{1} \cos \theta+\hat{a}_{2} \sin \theta, \\
& \hat{b}_{\theta}=\hat{b}_{1} \cos \theta+\hat{b}_{2} \sin \theta .
\end{aligned}
$$

In the high squeezing regime, the fluctuations in the joint quadratures, $\hat{a}_{1}-\hat{b}_{1}$ and $\hat{a}_{2}+\hat{b}_{2}$, are simultaneously well below the vacuum level. This is the experimental signature of the EPR entanglement. This does not violate Heisenberg's uncertainty principle because $\left[\hat{a}_{1}-\hat{b}_{1}, \hat{a}_{2}+\hat{b}_{2}\right]=0$. In analogy to the original EPR paper [12,15], $\hat{a}_{-\theta}$ is maximally correlated with $\hat{b}_{\theta}$. This correlation allows us to reduce our

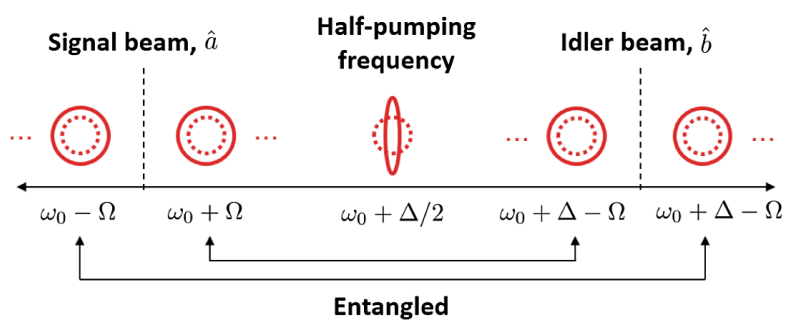

FIG. 2. Visualization of the frequency-mode entanglement in the pumped optical parametric amplifier in our proposed scheme. 
uncertainty in $\hat{a}_{-\theta}$ by making a measurement on $\hat{b}_{\theta}$. This key theoretical result that enables our conditional squeezing scheme has recently been realized experimentally [16].

\section{B. Interferometer as detector and filter}

The signal and idler beams enter the dark port of the interferometer and couple to the laser that enters the bright port. The interferometer we consider has both signal and power recycling cavities to increase sensitivity as shown in Fig. 1. To the signal beam, the interferometer looks like a resonant cavity. The input-output relation for the phase quadrature of the signal beam (which will contain our GW signal) is [7]

$$
\hat{A}_{2}=e^{2 i \beta}\left(\hat{a}_{2}-\mathcal{K} \hat{a}_{1}\right)+\sqrt{2 \mathcal{K}} \frac{h}{h_{\mathrm{SQL}}} e^{i \beta},
$$

where $h_{\mathrm{SQL}}=\sqrt{8 \hbar /\left(m \Omega^{2} L_{\mathrm{arm}}^{2}\right)}$ is the square root of the SQL, $\beta$ is a phase shift given as $\beta \equiv \arctan \Omega / \gamma$, with $\gamma$ being the detection bandwidth, and $\mathcal{K}$ is the optomechanical coupling constant that determines the coupling between the light and the interferometer mirrors. For current GW detectors, the signal-recycling cavity length is of the order of 10 meters, and the SRM transmission is quite high (tens of percent). In this case, we can effectively view the signal-recycling cavity (SRC) formed by SRM and ITM as a compound mirror by ignoring the propagation phase $\Omega L_{\mathrm{SRC}} / c$ picked up by the sidebands, as first mentioned by Buonanno and Chen [17]. When the SRC is tuned, the corresponding optomechanical coupling constant $\mathcal{K}$ is the same as that given by Kimble et al. [7]:

$$
\mathcal{K}_{\mathrm{KLMTV}}=\frac{32 \omega_{0} P_{\mathrm{arm}}}{m L_{\mathrm{arm}}^{2} \Omega^{2}\left(\Omega^{2}+\gamma^{2}\right)},
$$

where $P_{\text {arm }}$ is the arm cavity power, $m$ is the mirror mass, and $\gamma$ is equal to $c T_{\mathrm{SRC}} /\left(4 L_{\mathrm{arm}}\right)$, with $T_{\mathrm{SRC}}$ being the effective power transmission of the SRC when viewed as a compound mirror.

For LBIs, the definition of such a coupling constant can differ from that given by Kimble et al. This is because the SRC length is longer and also because the SRM transmission becomes comparable to that of ITM in order to broaden the detection bandwidth in the resonant-sidebandextraction mode; i.e., the effective transmissivity $T_{\mathrm{SRC}}$ of SRC approaches 1. The approximation for defining $\gamma$ applied in Ref. [17], which assumes $T_{\mathrm{SRC}} \ll 1$, starts to break down. We also need to take into account the frequency-dependent propagation phase of the sidebands, which leads to the following expression for the coupling constant [18]:

$$
\mathcal{K}_{\mathrm{LBIs}}=\frac{2 h_{\mathrm{SQL}}^{2} L_{\mathrm{arm}} \omega_{0} P_{\mathrm{arm}} \gamma_{s} \omega_{s}^{2}}{\hbar c\left[\gamma_{s}^{2} \Omega^{2}+\left(\Omega^{2}-\omega_{s}^{2}\right)^{2}\right]} .
$$

Here $L_{\text {arm }}$ is the interferometer arm length; $\omega_{s}$ is a resonant frequency that arises from the coupling between the signal recycling and arm cavities. The frequency and bandwidth for such a resonance are given by

$$
\omega_{s}=\frac{c T_{\mathrm{ITM}}}{2 \sqrt{L_{\mathrm{arm}} L_{\mathrm{SRC}}}}, \quad \gamma_{s}=\frac{c T_{\mathrm{SRM}}}{4 L_{\mathrm{SRC}}} .
$$

The idler beam, however, is far away from the carrier frequency and does not produce a noticeable radiation pressure effect on the test mass. As such, it sees the interferometer as a simple detuned cavity as shown in Fig. 3, the same as was done in Ref. [17]. As such, the idler beam simply experiences a frequency-dependent ellipse rotation. This can be seen in the idler input-output relation which is given as

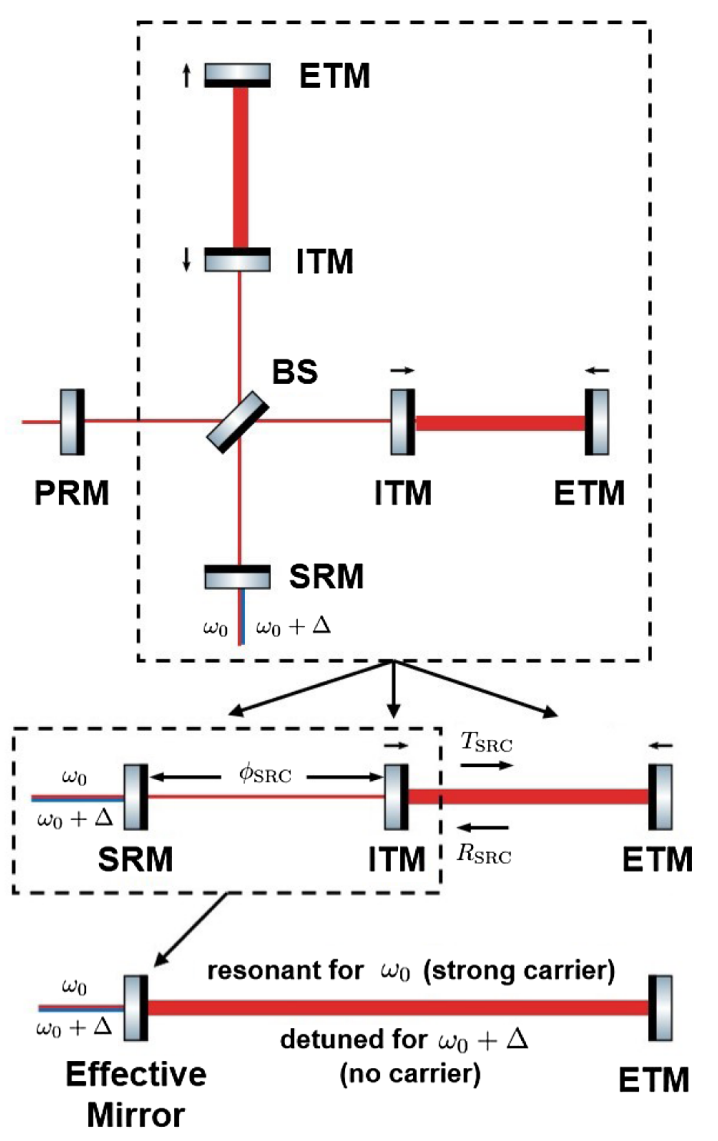

FIG. 3. The signal recycling interferometer can be mapped to a three-mirror cavity. The signal recycling cavity can then be mapped into a single mirror with effective transmissivities and reflectivities [17]. This final, two-mirror cavity is resonant for the signal beam (at $\omega_{0}$ ) but detuned for the idler beam (at $\omega_{0}+\Delta$ ); thus, the idler simply experiences a frequency-dependent ellipse rotation. This allows us to use the interferometer itself as a filter cavity. The single-trip propagation phase $\phi_{\mathrm{SRC}}$ is equal to the integer number of $\pi$ for the carrier in the resonant-sidebandextraction mode, and it is equal to some specific number for the idler, as explained in the text. 


$$
\hat{B}_{2}=e^{i \alpha}\left(-\hat{b}_{1} \sin \Phi_{\mathrm{rot}}+\hat{b}_{2} \cos \Phi_{\mathrm{rot}}\right),
$$

where $\alpha$ is an unimportant overall phase and the important rotation angle $\Phi_{\text {rot }}$ is given by $[7,11]$

$$
\Phi_{\mathrm{rot}}=\arctan \left(\frac{\Omega+\delta_{f}}{\gamma_{f}}\right)+\arctan \left(\frac{-\Omega+\delta_{f}}{\gamma_{f}}\right) .
$$

Here $\delta_{f}$ and $\gamma_{f}$ are the effective detuning and bandwidth of the interferometer with respect to the idler beam. They are defined as

$$
\begin{gathered}
2\left(\omega_{\text {idler }}+\delta_{f}\right)\left(L_{\text {arm }} / c\right)+\arg \left(r_{\text {SRC }}^{\text {idler }}\right)=2 n \pi, \\
\gamma_{f} \equiv c\left|t_{\mathrm{SRC}}^{\text {idler }}\right|^{2} /\left(4 L_{\text {arm }}\right),
\end{gathered}
$$

where $\omega_{\text {idler }}=\omega_{0}+\Delta, n$ is an integer number, and $r_{\mathrm{SRC}}^{\text {idler }}$ and $r_{\mathrm{SRC}}^{\text {idler }}$ are the effective amplitude reflectivity and transmissivity of the SRC for the idler beam:

$$
\begin{gathered}
r_{\mathrm{SRC}}^{\text {idler }}=\sqrt{R_{\mathrm{ITM}}}+\frac{T_{\mathrm{ITM}} \sqrt{R_{\mathrm{SRM}}}}{1-\sqrt{R_{\mathrm{ITM}} R_{\mathrm{SRM}}} e^{2 i} \phi_{\mathrm{SRC}}^{\text {idler }}}, \\
t_{\mathrm{SRC}}^{\text {idler }}=\frac{\sqrt{T_{\mathrm{SRM}} T_{\mathrm{ITM}}} e^{i \phi_{\mathrm{SRC}}^{\text {ider }}}}{1-\sqrt{R_{\mathrm{ITM}} R_{\mathrm{SRM}}} e^{2 i \phi_{\mathrm{SRC}}^{\text {ider }}}} .
\end{gathered}
$$

The phase $\phi_{\mathrm{SRC}}^{\text {idler }}=\Delta L_{\mathrm{SRC}} / c$ by assuming $\omega_{0} L_{\mathrm{SRC}} / c$ is equal to the integer number of $\pi$ as in the resonantsideband-extraction mode. Note that the issue of the compound-mirror approximation for the carrier mentioned earlier does not occur for the idler beam. Because $\Delta \gg \Omega$, the sideband propagation phase inside SRC can be ignored, and also the effective SRC transmissivity for the idler $T_{\mathrm{SRC}}^{\mathrm{idler}}=\left|t_{\mathrm{SRC}}^{\mathrm{idler}}\right|^{2}$ is much smaller than 1 , which makes $\gamma_{f}$ properly defined.

The rotation angle $\Phi_{\text {rot }}$ needs to be equal to $\arctan \mathcal{K}$ to achieve the required frequency-dependent squeezing. This usually cannot be realized exactly with a single cavity, and two cavities are required. Indeed, for LIGO implementation of such an idea [8], the rotation in the intermediate frequency deviates from the ideal one by a noticeable amount. As we will see, for LBIs, the broadband operation mode can make such a deviation negligible because the transition frequency from the radiation-pressure-noise dominant regime to the shot-noise dominant regime is much lower than the detection bandwidth; a single filter cavity is close to being sufficient and ideal [19]. The corresponding required detuning and bandwidth given in Eq. (9) and $\omega_{s} \gg \Omega$, following a similar derivation as Refs. [8,19], are

$$
\begin{gathered}
\gamma_{f}=\left.\sqrt{\frac{\Omega^{2} \mathcal{K}_{\mathrm{LBIs}}}{2}}\right|_{\omega_{s} \gg \Omega} \approx \sqrt{\frac{4 \omega_{0} P_{\mathrm{arm}} T_{\mathrm{SRM}}}{m c^{2} T_{\mathrm{ITM}}^{2}}}, \\
\delta_{f}=-\gamma_{f} .
\end{gathered}
$$

From Eq. (46) in [7], one can show that the sensitivity of the interferometer with an imperfect rotation angle is

$S_{h} \approx \frac{h_{\mathrm{SQL}}^{2}}{2 \cosh 2 r}\left(\mathcal{K}+\frac{1}{\mathcal{K}}\right)+\frac{h_{\mathrm{SQL}}^{2}}{2} \frac{\sinh ^{2} 2 r}{\cosh 2 r}\left(\mathcal{K}+\frac{1}{\mathcal{K}}\right) \delta \Phi^{2}$

where $r$ is the squeezing factor and $\delta \Phi=\Phi_{\text {rot }}-$ $\arctan \mathcal{K} \ll 1$. The first term in Eq. (19) is the sensitivity when the rotational angle is realized exactly and the second term is the degradation in sensitivity due to the error in the rotational angle. In the case of a $15-\mathrm{dB}$ squeezing injection as considered in [8], $r=1.73$, so the ratio of the correction term to the exact term is $\approx 249 \delta \Phi^{2}$. So, if we want to keep the relative correction to less than $10 \%$, we will need the error in the rotation angle $\delta \Phi<0.02 \mathrm{rad}$ [i.e., $249(0.02 \mathrm{rad})^{2} \times$ $100 \%=9.96 \%<10 \%]$. So, as long as the proposed scheme keeps the overall error in the rotation angle to less than $0.02 \mathrm{rad}$, we will suffer no more than a $10 \%$ degradation in noise reduction. This requirement turns out to be easily satisfied in the broadband detection mode of LBIs due to the reason mentioned earlier.

\section{NUMERICAL RESULTS}

In this section, we show the systematic approach to finding the working points for implementing this idea. The procedure was outlined in Ref. [8], which showed one working point out of many for LIGO parameters. The tunable parameters are the detuning frequency of the pump $\Delta$, the small change of the arm length $\delta L_{\text {arm }}$, and the SRC length $\delta L_{\mathrm{SRC}}$ with respect to their macroscopic values. We show how the relevant domains for the various tunable parameters in our scheme were derived. Then, we present the result of our search for solutions to the resonance condition within these bounds.

For illustration, we choose the detector parameters on a scale similar to the Einstein Telescope. To highlight that the EPR squeezing scheme is not restricted to one particular set of detector design parameters, we allow the macroscopic arm length $L_{\text {arm }}$ and $L_{\mathrm{SRC}}$ to vary from the nominal values outlined in the Einstein Telescope design study [3]. The detector parameters are outlined in the following table.

\begin{tabular}{lcc}
\hline Parameters & Name & Value \\
\hline$L_{\text {arm }}$ & Arm cavity length & {$[9995,10005] \mathrm{m}$} \\
$L_{\text {SRC }}$ & Signal recycling cavity length & {$[100,200] \mathrm{m}$} \\
$m$ & $\quad$ Mirror mass & $150 \mathrm{~kg}$ \\
$T_{\text {ITM }}$ & ITM power transmissivity & 0.04 \\
$T_{\text {SRM }}$ & SRM power transmissivity & 0.04 \\
$P_{\text {arm }}$ & Intracavity power & $3 \mathrm{MW}$ \\
$r$ & Squeezing parameter & $1.73(15 \mathrm{~dB})$ \\
\hline
\end{tabular}

To start, we equate Eq. (14) with Eq. (17) and solve for $\phi_{\text {SRC }}$. This is the exact phase accumulated by the idler after 
one round trip in the SRC, so we denote it $\phi_{\mathrm{SRC}}^{\text {exact }}$. Next, for the effective cavity to have a detuning frequency satisfying Eq. (18), $\delta_{f}=-\gamma_{f}$, we tune the idler detuning $\Delta, \delta L_{\text {arm }}$, and $\delta L_{\mathrm{SRC}}$ to find solutions to Eq. (13). The idler detuning $\Delta$ has to be in the low $\mathrm{MHz}$ regime because if it was lower it would interfere with the carrier, but if it were too high, electronics would not work optimally. The allowable range is taken to be

$$
\frac{\Delta}{2 \pi} \in[5,50] \mathrm{MHz}
$$

Since we want to keep $\gamma_{f}$ fixed while we tune $\Delta$ to make the resonance condition Eq. (13) satisfied for $\delta_{f}=-\gamma_{f}$, we can only alter $\Delta$ by integer numbers $n$ of the free spectral range of the SRC, namely, $\Delta=\left(\phi_{\mathrm{SRC}}^{\text {exact }}+n \pi\right) c / L_{\mathrm{SRC}}$. The minimum allowed detuning is $5 \mathrm{MHz}$, and this will occur when $L_{\text {SRC }}$ is at its maximum, i.e., $200 \mathrm{~m}$. This will correspond to the minimum allowed $n$. Similarly, the max detuning occurs at the minimum SRC length and the $\max n$. We find the relevant values of $n$ to be

$$
n \in[7,33] .
$$

We find that the overall rotation angle $\Phi_{\text {rot }}$ is not very sensitive to changes in the SRC phase. It is acceptable to have $\phi_{\mathrm{SRC}}$ slightly deviated from the exact value $\phi_{\mathrm{SRC}}^{\text {exact }}$. This makes it easier for us to find solutions to our resonance condition Eq. (13). As noted before, we must keep the error in the overall rotation angle to less than $0.02 \mathrm{rad}$. That is, we need $|\delta \Phi|=\left|\Phi_{\text {rot }}-\arctan \mathcal{K}\right|<0.02 \mathrm{rad}$ where $\Phi_{\text {rot }}$ is given in Eq. (12). To ensure the error in rotation angle is less than $0.02 \mathrm{rad}$ over the whole positive frequency domain, we require

$$
\max _{\Omega}\left|\Delta \phi_{\mathrm{SRC}} \frac{d \Phi_{\mathrm{rot}}}{d \phi_{\mathrm{SRC}}}\right|<0.02
$$

Using the parameters listed in the table, one finds

$$
\left|\Delta \phi_{\mathrm{SRC}}\right|<0.002
$$

To show the working points for different macroscopic arm lengths and SRC lengths, we scan the length by 1-m step size. Additionally, we sweep $\phi_{\text {SRC }}^{\text {appox }}$ in between $\left[\phi_{\mathrm{SRC}}^{\text {exact }}-0.002, \phi_{\mathrm{SRC}}^{\text {exact }}+0.002\right]$ with a step size of 0.0001 . There are 1.2 million combinations of these parameters with the given step sizes. We take advantage of the fact that each combination is independent and can thus be checked in parallel. We define the working points as those that require microscopic changes of arm length $\Delta L_{\text {arm }}$ and SRC length $\delta L_{\mathrm{SRC}}$ smaller than $1 \mathrm{~cm}$. Our search resulted in 3444 working points summarized in Fig. 5. We pick one of

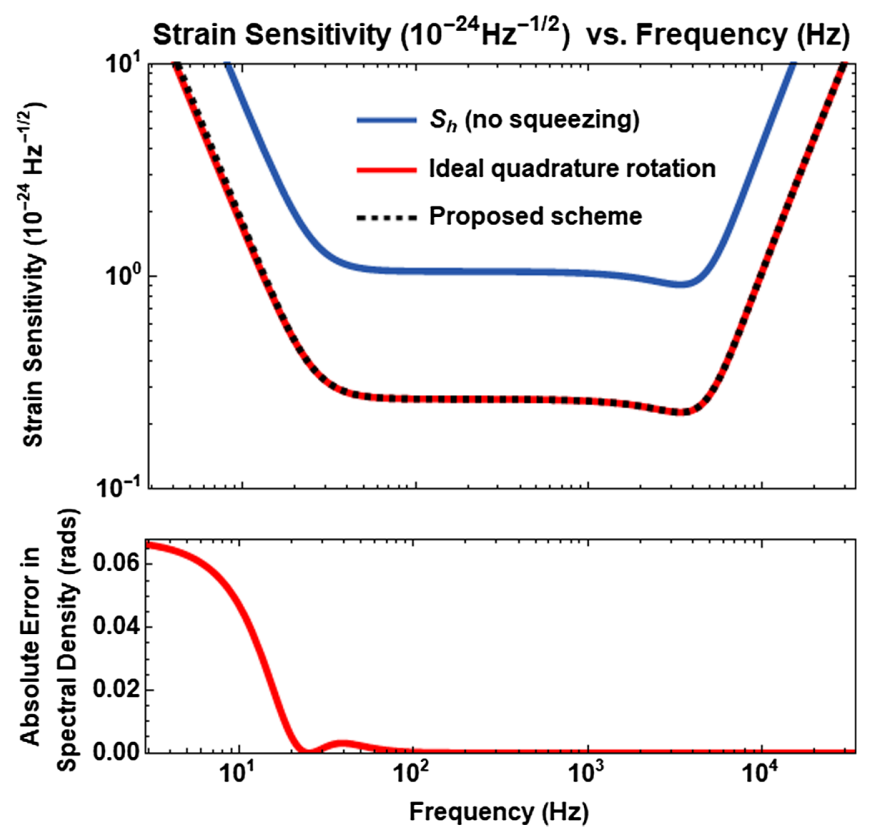

FIG. 4. Approximate sensitivity (dotted curve) plotted alongside the sensitivity when ideal ellipse rotation is achieved (red curve). For this plot, we use $L_{\text {arm }}=10003 \mathrm{~m}, L_{\mathrm{SRC}}=100 \mathrm{~m}$, $\Delta /(2 \pi)=1.04 \mathrm{MHz}$, and $\phi_{\mathrm{SRC}}^{\mathrm{approx}}=0.16828$.

the many working points for illustration, and the resulting sensitivity curve is given in Fig. 4. The EPR scheme achieves almost the ideal frequency-dependent rotation of the squeezing quadrature angle. This is the result of us heavily restricting our parameter space to bound the error.

The step sizes were chosen with computational expenses in mind, so the resolution is not particularly high. As such, Fig. 5 shows several "dead zones" as well as a couple of "hot points." The natural question to ask is whether these are real or whether they are a by-product of our numerical precision. Zooming in around two such points, we produced the subfigures on the right-hand side of Fig. 5. In the case of the dead zone, we see that there are actually working points where there appeared to be none. This is promising, as it points to the conclusion that a working point can be found given precise fine-tuning. Similarly, we zoomed in on a hot point (top right panel of Fig. 5), and interestingly we still see a line structure where there are as many as 35 working points surrounded by areas that apparently have zero working points. So, to check whether this was a real feature of the system or an issue of numerical precision, we again zoomed in around the hot points. What we found, once again, was that the dead zones must simply be due to the numerical precision chosen. With more time or computational power (or both), one could map a relatively smooth landscape of working points for the ET. In our case, our goal was to simply show that this EPR-based squeezing scheme is not very sensitive to the actual arm length and SRC length, and that we can always find some working points for given a set of parameters. 


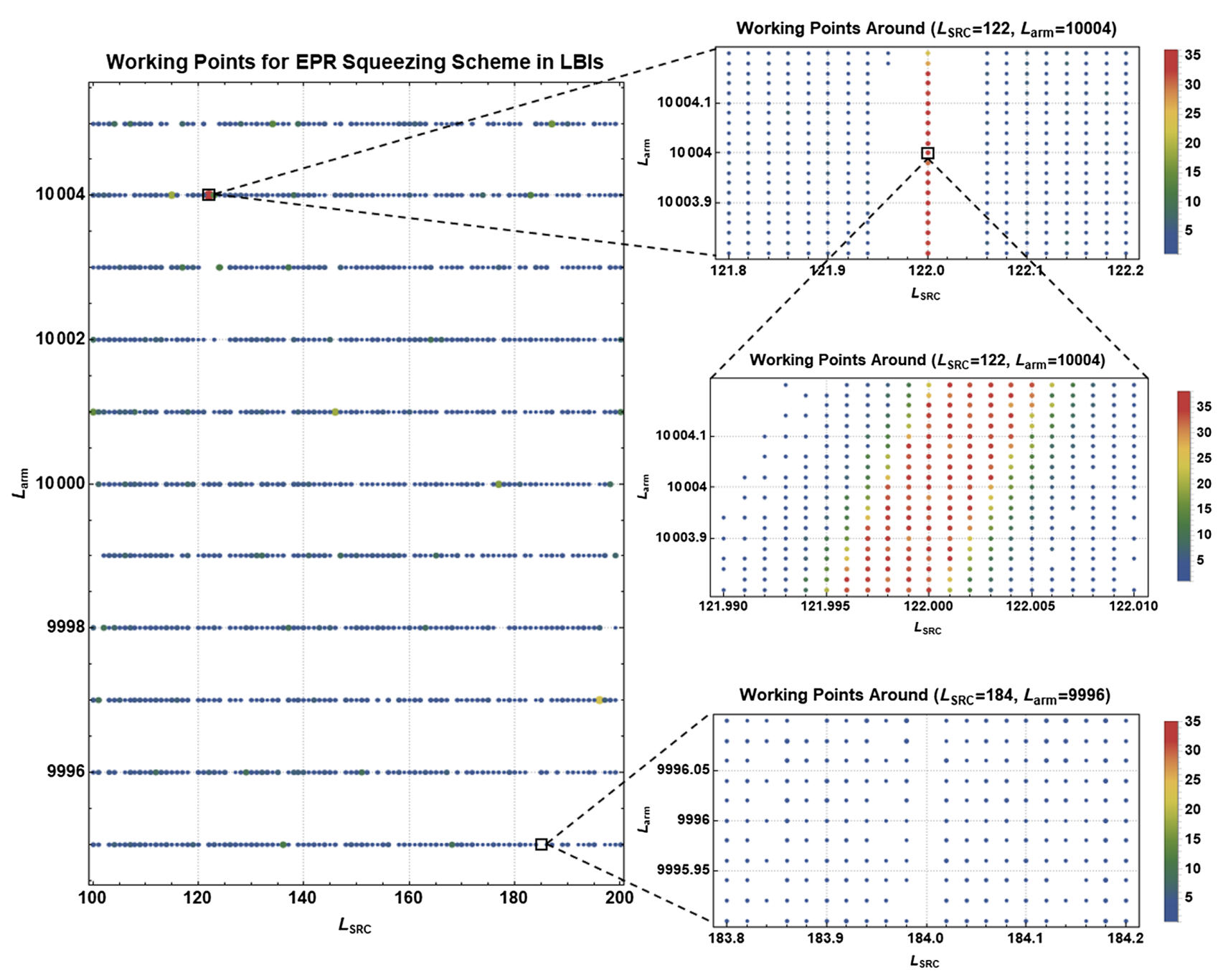

FIG. 5. The left panel shows all the working points found at the resolution set by the step size of the arm cavity length and SRC length to be $1 \mathrm{~m}$. The color of the point indicates how many working points exist at a given combination of $L_{\mathrm{SRC}}$ and $L_{\mathrm{arm}}$. The right column of plots are zoomed in considerably. The existence of working points in these plots indicates that with sufficient fine-tuning, working points for this squeezing scheme can always be found.

\section{CONCLUSIONS}

We have shown that EPR entanglement-based squeezing can be implemented in LBIs. We derived the relevant bounds on the tunable parameters to ensure that our approximate ellipse rotation scheme very nearly matches the exact rotation achievable through the use of external filter cavities. The goal of the project was to map the interferometer working points for this squeezing scheme in LBIs like the ET and Cosmic Explorer. We accomplished this at a rather low resolution of the parameter space. Zooming in around areas that had very many working points or very few showed that the landscape of working points seems to be quite smooth. In other words, if an area appears to have no working points, it is likely because the step size used to iterate through the parameter space was too low. This is ideal for experimental implementation of such a scheme, for if we cannot fulfill the requirement given the nominal parameters, there should be another working point less than a few centimeters away. As such, we conclude that EPR-based squeezing is an appealing alternative to other broadband quantum noise reduction schemes that require additional filter cavities.

\section{ACKNOWLEDGMENTS}

We would like to thank P. Jones and A. Freise for fruitful discussions. J. B. was supported by a Fulbright-University of Birmingham postgraduate grant during the completion of this research. H. M. has been supported by the UK STFC Ernest Rutherford Grant No. ST/M005844/11. 
[1] LIGO Scientific Collaboration and Virgo Collaborations, Observation of Gravitational Waves from a Binary Black Hole Merger, Phys. Rev. Lett. 116, 061102 (2016).

[2] LIGO Scientific Collaboration and Virgo Collaboration, GW170817: Observation of Gravitational Waves from a Binary Neutron Star Inspiral, Phys. Rev. Lett. 119, 161101 (2017).

[3] ET Science Team, Einstein Gravitational Wave Telescope Conceptual Design Study http://www.et-gw.eu/index.php/ etdsdocument (2019).

[4] B. P. Abbott et al., Exploring the sensitivity of next generation gravitational wave detectors, Classical Quantum Gravity 34, 044001 (2017).

[5] C. M. Caves, Quantum-mechanical noise in an interferometer, Phys. Rev. D 23, 1693 (1981).

[6] R. Schnabel, Squeezed states of light and their applications in laser interferometers, Phys. Rep. 684, 1 (2017).

[7] H. J. Kimble, Y. Levin, A. B. Matsko, K. S. Thorne, and S. P. Vyatchanin, Conversion of conventional gravitationalwave interferometers into quantum nondemolition interferometers by modifying their input and/or output optics, Phys. Rev. D 65, 022002 (2001).

[8] Y. Ma, H. Miao, B. H. Pang, M. Evans, C. Zhao, Jan Harms, R. Schnabel, and Y. Chen, Proposal for gravitational-wave detection beyond the standard quantum limit through EPR entanglement, Nat. Phys. 13, 776 (2017).

[9] J. Südbeck, S. Steinlechner, M. Korobko, and R. Schnabel, Demonstration of interferometer enhancement through EPR entanglement, arXiv:1908.09602.

[10] M. J. Yap, P. Altin, T. G. McRae, R. L. Ward, B. J. J. Slagmolen, and D. E. McClelland, Generation and control of frequency-dependent squeezing via EPR entanglement, arXiv:1908.08685.
[11] D. D. Brown, H. Miao, C. Collins, C. Mow-Lowry, D. Töyrä, and A. Freise, Broadband sensitivity enhancement of detuned dual-recycled michelson interferometers with EPR entanglement, Phys. Rev. D 96, 062003 (2017).

[12] M. D. Reid, Demonstration of the Einstein-Podolsky-Rosen paradox using nondegenerate parametric amplification, Phys. Rev. A 40, 913 (1989).

[13] Z. Y. Ou, S.F. Pereira, H. J. Kimble, and K. C. Peng, Realization of the Einstein-Podolsky-Rosen Paradox for Continuous Variables, Phys. Rev. Lett. 68, 3663 (1992).

[14] C. M. Caves and B. L. Schumaker, New formalism for twophoton quantum optics. I. Quadrature phases and squeezed states, Phys. Rev. A 31, 3068 (1985).

[15] A. Einstein, B. Podolsky, and N. Rosen, Can quantummechanical description of physical reality be considered complete?, Phys. Rev. 47, 777 (1935).

[16] W. Li, Y. Jin, X. Yu, and J. Zhang, Enhanced detection of a low-frequency signal by using broad squeezed light and a bichromatic local oscillator, Phys. Rev. A 96, 023808 (2017).

[17] A. Buonanno and Y. Chen, Scaling law in signal recycled laser-interferometer gravitational-wave detectors, Phys. Rev. D 67, 062002 (2003).

[18] D. Martynov, H. Miao, H. Yang, F. H. Vivanco, E. Thrane, R. Smith, P. Lasky, W. E. East, R. Adhikari, A. Bauswein et al., Exploring the sensitivity of gravitational wave detectors to neutron star physics, Phys. Rev. D 99, 102004 (2019).

[19] F. Y. Khalili, Quantum variational measurement in the next generation gravitational-wave detectors, Phys. Rev. D 76, 102002 (2007). 